\title{
Universal PCR primers for ribosomal protein gene introns of fish
}

\author{
Seinen Chow $\cdot$ Takashi Yanagimoto
}

Received: 5 November 2015/Accepted: 5 January 2016/Published online: 28 January 2016

(C) The Author(s) 2016. This article is published with open access at Springerlink.com

\begin{abstract}
Human ribosomal protein (RP) gene sequences with respect to intron/exon structures and corresponding cDNA or genomic data of fish species were obtained from the GenBank database. Based on conserved exon sequences, 128 primer pairs for 41 genes were designed for exon-primed intron-crossing (EPIC) polymerase chain reaction (PCR). In reference to the draft genome sequences of the Pacific bluefin tuna (Thunnus orientalis), 12 primer pairs expected to amplify introns of the bluefin tuna with lengths of 500-1000 bp were selected and applied to six distantly related fish species belonging to the Orders Clupeiformes, Tetraodontiformes, Pleuronectiformes, Perciformes, Scorpaeniformes, and Anguilliformes. PCR amplification was observed for at least four species in each primer pair, and all fragments were larger than those expected for intronless amplification. Single fragment amplification was observed for at least seven primer pairs per species. Fragment sizes of the bluefin tuna for nine primer pairs corresponded to those expected from the genomic data. Thus, our primer pairs are potentially applicable to a wide variety of fish species and serve as an initial step for isolating single-copy nuclear DNA sequences.
\end{abstract}

Keywords Universal primers $\cdot$ Ribosomal protein genes $\cdot$ Intron $\cdot$ Single copy nuclear loci $\cdot$ Teleostei

\section{Introduction}

Because introns are a good source to find DNA polymorphisms in the eukaryotic genome, exon-primed introncrossing (EPIC) polymerase chain reaction (PCR) assays have been developed for genetic analyses (Lessa 1992; Palumbi and Baker 1994; Corte-Real et al. 1994; Chow and Hazama 1998; Chow 1998; Quattro and Jones 1999; Hassan et al. 2002; Jarman et al. 2002; Chow and Nakadate 2004; Nakadate and Chow 2008; Pinho et al. 2010; Jennings and Etter 2011; Chow et al. 2015). Polymorphisms detected in introns have been used for genetic population analysis (Palumbi and Baker 1994; Chow and Takeyama 2000; Williams et al. 2002; Nohara et al. 2003; Touriya et al. 2003; Nakadate et al. 2005; Berrebi et al. 2005; Atarhouch et al. 2007; Chow et al. 2007; Yanagimoto et al. 2012), investigations of interspecific hybridization (Daguin et al. 2001; Pacheco et al. 2002; Yao et al. 2015), and phylogenetic analysis (DeBry and Seshadri 2001; Berrebi et al. 2005; Creer 2007; Near and Cheng 2008; He et al. 2008; Pinho et al. 2010).

The universal specificity of primers may depend on the conservation of exon sequences whereas the efficiency of isolating a single copy of the target nuclear genome may be a function of the number of duplicate

S. Chow $(\bowtie) \cdot$ T. Yanagimoto

Research Center for Aquatic Genomics, National Research Institute of Fisheries Science, 2-12-4 Fukuura, Kanazawa,

Yokohama, Kanagawa 236-8648, Japan

e-mail: chow@affrc.go.jp 
gene copies, paralogs, and/or pseudogenes. Ribosomal proteins (RPs), which play fundamental roles in protein synthesis, are the most highly conserved proteins among eukaryotes, and each protein is typically encoded by a single gene (Wool 1979; Wool et al. 1995; Kenmochi et al. 1998; Yoshihama et al. 2002). Therefore, RP genes may be particularly suitable for designing universal primers. In spite of this potential, the number of studies reporting universal primers for the amplification of RP gene introns of aquatic animals is small (Chow and Hazama 1998; Pinho et al. 2010; Chow et al. 2015). Here we report the development of universal primer pairs to amplify 128 intron regions of 41 RP genes in fishes, which may be potentially applicable to distantly related fish species.

\section{Materials and methods}

The intron/exon structures of human RP genes have been reported by Kenmochi et al. (1998) and Yoshihama et al. (2002), and the nucleotide sequences are available in the GenBank database (http://www.ncbi.nlm.nih. gov/genbank/). Reference cDNA data of Danio rerio, Ictalurus punctatus, Salmo salar, Takifugu rubripes, and Paralichthys olivaceus, corresponding to orthologs of the human RP genes were obtained from the GenBank database. These fish species are taxonomically distant one another and offer abundant data set of ribosomal protein gene. The Orders to which these fish species belong were Cypriniformes, Siluriformes, Salmoniformes, Tetraodontiformes, and Pleuronectiformes, respectively, and we anticipated that primers designed using conserved sequences among these distant fish species may have a universal specificity. The draft genome of the Pacific bluefin tuna, Thunnus orientalis (accession nos. BADN01000001-BADN01133062 in the DNA Data Bank of Japan) (Nakamura et al. 2013) was also utilized. The Pacific bluefin tuna belongs to the Order Perciformes. Multiple sequence alignments were performed using ClustalX version 1.83 (Thompson et al. 1997), and conserved exon regions were visually inspected. Rules for primer design included; primer length of 20 nucleotides, less than two degenerate bases, a moderate GC content (approximately $50 \%$ ), no degenerated bases within last two positions, and avoiding presence of the primer secondary structure produced by inter- and intra-molecular interactions. Furthermore, more than $20 \mathrm{bp}$ distances was placed between the splicing site and $3^{\prime}$-end of at least one of a primer in each pair, which may be used to authenticate amplicons by nucleotide sequence analysis. All these procedures were performed by eyes.

Because intron size cannot be predicted and there may be considerable variations even between closely related taxa, draft genome sequences of the Pacific bluefin tuna were used to select primer pairs for PCR testing. A total of 12 primer pairs expected to amplify RP gene introns of the Pacific bluefin tuna with lengths of 500-1000 bp were selected for ease of sizing, and PCR amplification was attempted using template DNA of the Japanese pilchard (Sardinops melanostictus), the Japanese puffer (Takifugu rubripes), starry flounder (Platichthys stellatus), the Pacific bluefin tuna (T. orientalis), broadbanded thornyhead (Sebastolobus macrochir), and the Japanese eel (Anguilla japonica), all derived from the tissue collection in our laboratory. These fishes belong to the Orders Clupeiformes, Tetraodontiformes, Pleuronectiformes, Perciformes, Scorpaeniformes, and Anguilliformes, respectively, and used to evaluate universal specificity of the primer pairs selected. PCR amplification was carried out in a $12 \mu \mathrm{L}$ final volume containing $1 \mu \mathrm{L}$ of template DNA $(10-50 \mathrm{ng} / \mu \mathrm{L}$ ), $1.2 \mu \mathrm{L}$ of $10 \times$ reaction buffer (containing $20 \mathrm{mM} \mathrm{MgCl}$ ), $1 \mathrm{mM}$ of each deoxynucleotide triphosphate, $0.4 \mu \mathrm{M}$ of each primer, and 0.5 U of EX Taq polymerase (Takara Bio, Inc., Shiga, Japan). The same reaction conditions were used for all primer pairs, in which the reaction mixtures were preheated to $94{ }^{\circ} \mathrm{C}$ for $4 \mathrm{~min}$, followed by 35 amplification cycles (denaturing at $94{ }^{\circ} \mathrm{C}$ for $30 \mathrm{~s}$, annealing at $56{ }^{\circ} \mathrm{C}$ for $30 \mathrm{~s}$, and extension at $72{ }^{\circ} \mathrm{C}$ for $50 \mathrm{~s}$ ), with a final extension at $72{ }^{\circ} \mathrm{C}$ for $7 \mathrm{~min}$. PCR products were electrophoresed on $1.5 \%$ agarose gels (UltraPure Agarose; Invitrogen Corporation, Carlsbad, CA, USA) and stained with ethidium bromide. The gel images visualized on transilluminator were captured by CCD camera.

\section{Results and discussion}

Based on the relatively high degree of conservation of the exon sequences among distant reference fish species and human, a total of 128 primer pairs for the amplification of 41 RP genes were designed for EPIC PCR. Among the 41 RP genes listed in Table 1, whole or partial sequences of 29 genes (Table 1, dagger) were 
Table 1 Sequences of 128 primer pairs for 41 ribosomal protein (RP) genes

\begin{tabular}{|c|c|c|c|c|c|c|c|}
\hline RP & Pair & Forward & $5^{\prime}-3^{\prime}$ & $T m$ & Reverse & $5^{\prime}-3^{\prime}$ & $T m$ \\
\hline $\mathrm{L}^{\dagger}$ & 1 & L3ExAF & GGATACAARGCYGGCATGAC & 62.0 & L3ExBR & TCCACCACYTCCTTYTTGTT & 60.9 \\
\hline $\mathrm{L}^{\dagger}$ & 2 & L3ExBF & GCACRTCAGTGATGARTGCA & 63.5 & L3ExCR & TTGGTRAARGCCTTCTTCTT & 57.1 \\
\hline $\mathrm{L}^{\dagger}{ }^{\dagger}$ & 3 & L3ExCF & GGCAAGAARCAGCTGGAGAA & 64.3 & L3ExDR & CTCAGRGGCAGCAGRCGCAT & 68.1 \\
\hline $\mathrm{L}^{\dagger}{ }^{\dagger}$ & 4 & L3ExDF & CAGGAYGAGATGATYGACGT & 60.1 & L3ExER & AGCTTCTTBGTGTGCCAACG & 65.2 \\
\hline $\mathrm{L}^{\dagger}$ & $5^{*}$ & L8Ex2F & CAYATTGACTTCGCTGARCG & 60.8 & L8Ex3R & TTGCCGCAGTAGATRAACTG & 60.4 \\
\hline $\mathrm{L}^{\dagger}$ & 6 & L8Ex3F & CAGTTYATCTACTGCGGCAA & 60.4 & L8Ex4R & GGCTTCTCCTCCAGRCAGCA & 66.9 \\
\hline $\mathrm{L}^{\dagger}$ & 7 & L8Ex4F & АTCTCYTCTGCMAACAGAGC & 55.3 & L8Ex5R & TTCARGATGGGTTTGTCAAT & 60.2 \\
\hline $\mathrm{L}^{\dagger}$ & 8 & L8Ex5F & GACAAACCCATCYTGAAGGC & 63.4 & L8Ex6R & ATGTGCTGRTGGTTRCCACC & 62.3 \\
\hline $\mathrm{L} 9^{\dagger}$ & 9 & L9Ex2F & ATGAAGACYATTCTCAGYAA & 47.5 & L9Ex3R & GGTTRATGTGGTTGAACTCC & 58.3 \\
\hline $\mathrm{L} 9^{\dagger}$ & 10 & L9Ex3F & TTCAACCACATYAACCTGGA & 60.4 & L9Ex4R & CACCATTTWTCCACACGCAG & 63.9 \\
\hline $\mathrm{L} 9^{\dagger}$ & 11 & L9Ex4F & GTCCAGAACATGATYAAGGG & 58.4 & L9Ex5R & RAAGTTYCTGATCTCCACCA & 57.2 \\
\hline $\mathrm{L}^{\dagger}$ & 12 & L9Ex5F & CTGGTGGAGATCAGRAACTT & 58.3 & L9Ex6R & TTWGACACCWGCTCAATATC & 54.2 \\
\hline $\mathrm{L} 9^{\dagger}$ & 13 & L9Ex6F & GACGAGTTGRTTCTGGARGG & 61.2 & L9Ex7R & ACWGTGGTGGCYTGCTGGAT & 67.0 \\
\hline $\mathrm{L} 12^{\dagger}$ & 14 & L12ExAF & TTCATCCGACACCCTCACCA & 68.9 & L12ExBR & AGGTCCRATTTTGGGGGCCA & 71.3 \\
\hline $\mathrm{L} 12^{\dagger}$ & 15 & L12ExBF & TGGCCCCCAAAATYGGACCT & 71.3 & L12ExCR & AGGCCYTTCCAGTCACCGGT & 69.1 \\
\hline $\mathrm{L} 12^{\dagger}$ & 16 & $\mathrm{~L} 12 \mathrm{ExCF}$ & AAGCTGACCATCCAGAACAG & 61.9 & L12ExDR & TTCTTCCTGTCACGRGGAGG & 64.3 \\
\hline $\mathrm{L} 12^{\dagger}$ & $17 *$ & L12ExDF & CCTCCYCGTGACAGGAAGAA & 64.3 & L12ExER & CCCAGAATCTCCTTRATGGT & 59.4 \\
\hline $\mathrm{L} 17^{\dagger}$ & 18 & L17Ex2F & TCGCTCGACCCVGAGAACCC & 71.0 & L17Ex3R & TTAAAGTGRACACGAAGRTT & 53.9 \\
\hline $\mathrm{L} 17^{\dagger}$ & 19 & L17Ex3F & TCCAACCTTCGTGTYCACTT & 62.1 & L17Ex4R & TATGCATRCCYTTGATGGCCT & 63.0 \\
\hline $\mathrm{L} 17^{\dagger}$ & 20 & L17Ex4F & AAGCACCAGTGTGTYCCCTT & 63.5 & L17Ex5R & AGCATGTGVAGGAGGAACTC & 60.8 \\
\hline $\mathrm{L} 17^{\dagger}$ & 21 & L17Ex5F & CTBCACATGCTGAAGAACGC & 63.6 & L17Ex6R & AGAGAGTCCACATCYAAACC & 54.6 \\
\hline $\mathrm{L} 17^{\dagger}$ & 22 & L17Ex6F & AAGTYTCTCAGAAGAAAAAG & 51.9 & L17Ex7R & ATTTACTCCCGWGCCATAAG & 59.6 \\
\hline L18 & 23 & L18Ex3F & AATGCTCCCTTCAACARGGT & 62.4 & L18Ex4R & TGAATYCTGACATCATCAGT & 55.3 \\
\hline $\mathrm{L} 21^{\dagger}$ & 24 & $\mathrm{~L} 21 \mathrm{Ex} 2 \mathrm{~F}$ & CAGGCCCTTCCGCAAGCATG & 74.0 & L21Ex3R & CCYTTCTTRTAGATGCGCAT & 58.4 \\
\hline $\mathrm{L} 21^{\dagger}$ & 25 & L21Ex3F & ATGCGCATCTAYAAGAARGG & 58.4 & L21Ex4R & TAGCAYTTATGAGGCATRCC & 56.7 \\
\hline $\mathrm{L} 21^{\dagger}$ & $26^{*}$ & $\mathrm{~L} 21 \mathrm{Ex} 4 \mathrm{~F}$ & GTMGGCATCATTGTCAACAA & 62.0 & L21Ex5R & TCYCTGCTCTTTGAGTGCTT & 59.5 \\
\hline $\mathrm{L} 21^{\dagger}$ & 27 & L21Ex5F & AGCGTRTCAARGAGAACGAG & 58.7 & L21Ex6R & TTCTTRGTGCTGACGAAGTG & 58.2 \\
\hline $\mathrm{L} 24^{\dagger}$ & 28 & $\mathrm{~L} 24 \mathrm{Ex} 2 \mathrm{~F}$ & CTGTGCAGTTTYAGYGGGTA & 57.8 & L24Ex3R & AAGGCAGACTCRCAYTTGGC & 64.1 \\
\hline $\mathrm{L} 24^{\dagger}$ & 29 & L24Ex3F & ARCACAAGAAGGGCCAGTCT & 62.6 & L24Ex4R & СTTCTGGTTCCTCTTGGCCA & 66.5 \\
\hline $\mathrm{L} 24^{\dagger}$ & 30 & $\mathrm{~L} 24 \mathrm{Ex} 4 \mathrm{~F}$ & GCCAAGAGGAACCAGAAGCC & 67.2 & L24Ex5R & TCTTSGCCTCCTTKGCAGCC & 70.9 \\
\hline $\mathrm{L} 24^{\dagger}$ & 31 & L24Ex5F & GGCTGCMAAGGAGGCSAAGA & 70.9 & L24Ex6R & TTCATGGGCTTRGCRATCTT & 61.1 \\
\hline $\mathrm{L} 26^{\dagger}$ & 32 & $\mathrm{~L} 26 \mathrm{Ex} 2 \mathrm{~F}$ & TACAACGTGMGSTCCATGCC & 63.5 & L26Ex3R & TGCTGGCCTTTRTAGTGTCC & 61.6 \\
\hline $\mathrm{L} 26^{\dagger}$ & 33 & $\mathrm{~L} 26 \mathrm{Ex} 3 \mathrm{~F}$ & AACGGAACCACWGTCCAYG & 63.5 & $\mathrm{~L} 26 \mathrm{Ex} 4 \mathrm{R}$ & TCYTCCTTGTATTTGCCСTT & 60.1 \\
\hline $\mathrm{L} 27^{\dagger}$ & 34 & L27Ex2F & CTGGCTGGACGYTACKCCGG & 68.9 & L27Ex3R & TAAGGRCGGTCWGCGGTGCC & 71.6 \\
\hline $\mathrm{L} 27^{\dagger}$ & 35 & L27Ex3F & CHRCCATGGGCAAGAAGAAG & 64.4 & L27Ex4R & TTGTCCAGAGGAATRTCAAC & 56.5 \\
\hline $\mathrm{L} 27^{\dagger}$ & 36 & $\mathrm{~L} 27 \mathrm{Ex} 4 \mathrm{~F}$ & CCAAGGTCAAGTTTGAGGAG & 61.2 & L27Ex5R & CKGAGYTTCTGGAAGAACCA & 59.4 \\
\hline $\mathrm{L} 30^{\dagger}$ & 37 & L30Ex2F & AAAATGGTGGCCGCAAAGAA & 68.4 & L30Ex3R & TGGGACTGYTTGTAKCCCAG & 62.4 \\
\hline $\mathrm{L} 30^{\dagger}$ & $38 *$ & L30Ex3F & TCATCCTGGCCAACAACTGC & 68.5 & L30Ex4R & TTCCACTGTARTGRTGGACA & 58.9 \\
\hline $\mathrm{L} 30^{\dagger}$ & 39 & $\mathrm{~L} 30 \mathrm{Ex} 4 \mathrm{~F}$ & AAATACTWCAGGGTSTGCAC & 56.5 & L30Ex5R & GGCATRCTYCTGATGATGTC & 57.0 \\
\hline $\mathrm{L} 31^{\dagger}$ & 40 & L31Ex2F & AACRTSCACAAGCGCATCCA & 69.9 & L31Ex3R & RGGAGTKCCCATCTCCTTCA & 62.6 \\
\hline $\mathrm{L} 31^{\dagger}$ & 41 & L31Ex3F & TTCGCMRTGAAGGAGATGGG & 67.2 & L31Ex4R & CTCRTTRCGCTTCCTGGACA & 62.8 \\
\hline $\mathrm{L} 31^{\dagger}$ & 42 & $\mathrm{~L} 31 \mathrm{Ex} 4 \mathrm{~F}$ & CTGTCCAGGAAGCGYAAYGA & 62.8 & L31Ex5R & TCATCAACATTGACWGTCTG & 57.0 \\
\hline $\mathrm{L} 32^{\dagger}$ & 43 & L32Ex2F & AAGAGRACYAAGAAGTTCAT & 48.6 & L32Ex3R & GACYCTGTTRTCAATACCTC & 49.8 \\
\hline $\mathrm{L} 32^{\dagger}$ & 44 & L32Ex3F & AAGACCAARYACATGCTGCC & 61.5 & L32Ex4R & TTGTGRGCRATCTCTGCACA & 63.3 \\
\hline L34 & 45 & L34Ex2F & GCCTCCAACAARACYAGGCT & 62.1 & L34Ex3R & AGGTAYACRATGCGGTTACC & 57.9 \\
\hline L34 & 46 & L34Ex3F & TGCCCYGGYAGACTGCGTGG & 70.7 & L34Ex4R & TTKGTCTTTGARAGCCTCAT & 58.9 \\
\hline L34 & 47 & L34Ex4F & CCTATGGYGGTKCCATGTGT & 62.1 & L34Ex5R & TRAGGAAAGCACGCTTGATC & 61.8 \\
\hline $\mathrm{L}^{3} 6^{\dagger}$ & 48 & L36Ex3F & GCCATGGAGYTGCTGAARGT & 64.1 & $\mathrm{~L} 36 \mathrm{Ex} 4 \mathrm{R}$ & AGCYTTCCTCATGGCRGCCA & 69.3 \\
\hline
\end{tabular}


Table 1 continued

\begin{tabular}{|c|c|c|c|c|c|c|c|}
\hline $\mathrm{RP}$ & Pair & Forward & $5^{\prime}-3^{\prime}$ & $T m$ & Reverse & $5^{\prime}-3^{\prime}$ & $T m$ \\
\hline $\mathrm{L} 37^{\dagger}$ & $49^{*}$ & L37Ex2F & TCCWCCTGCGGCAAGTGTGG & 73.1 & L37Ex3R & TTGGCCTTRGCRCTCCAGTT & 63.6 \\
\hline $\mathrm{L} 37^{\dagger}$ & 50 & L37Ex3F & AACTGGAGTGCYAAGGCYAA & 58.2 & L37Ex4R & TGTTCCYTCACGGAAWCCAT & 64.2 \\
\hline $\mathrm{L} 39^{\dagger}$ & 51 & L39Ex1F & CWACYACCGCCATAGTGGTG & 62.9 & L39Ex2R & TTRATCCTGAARGTCTTGTG & 54.3 \\
\hline $\mathrm{L} 39^{\dagger}$ & 52 & L39Ex2F & GAARACTGGCAACAARATCA & 60.1 & L39Ex3R & CCCAGCTTGGTYCTTCTCCA & 66.5 \\
\hline $\mathrm{P}^{\dagger}$ & $53^{*}$ & P0ExAF & ATGATGCGYAARGCCATCCG & 66.8 & P0ExBR & GYAAGRTCCTCCTTGGTGAA & 58.1 \\
\hline $\mathrm{P}^{\dagger}$ & 54 & P0ExBF & TTYGTSTTCACCAAGGAGGA & 62.2 & P0ExCR & ATKGCWCCAGCACGGGCAGC & 74.8 \\
\hline $\mathrm{P}^{\dagger}$ & 55 & P0ExCF & GGGGMACCATTGAAATCYTG & 63.0 & P0ExDR & AGSAGCGTGGCYTCGCTGGC & 76.1 \\
\hline $\mathrm{P}^{\dagger}$ & 56 & P0ExDF & GGTGCTTGACATCACWGAGG & 63.4 & P0ExER & GGGTAGCCRATCTSCAGACA & 64.0 \\
\hline $\mathrm{P} 1^{\dagger}$ & 57 & P1Ex1F & TCTGTSTCCGARCTCGCCTG & 68.0 & $\mathrm{P} 1 \mathrm{Ex} 2 \mathrm{R}$ & AGAGCRTTCAGYTTGTCCTC & 58.4 \\
\hline $\mathrm{P} 1^{\dagger}$ & $58 *$ & $\mathrm{P} 1 \mathrm{Ex} 2 \mathrm{~F}$ & AAYGCYCTGATCAAGGCTGC & 64.4 & P1Ex3R & CAGATCAGRCTRCCGATGTC & 57.1 \\
\hline $\mathrm{P} 2^{\dagger}$ & 60 & $\mathrm{P} 2 \mathrm{Ex} 2 \mathrm{~F}$ & CGTTACGTKGCYGCTTACCT & 61.8 & P2Ex3R & TCTTTTCCATTTARYTCACT & 52.9 \\
\hline $\mathrm{P} 2^{\dagger}$ & 61 & $\mathrm{P} 2 \mathrm{Ex} 3 \mathrm{~F}$ & AAAGACATCRATGARGTCAT & 53.3 & $\mathrm{P} 2 \mathrm{Ex} 4 \mathrm{R}$ & ACAGCACCACCKGCYGGCAC & 71.2 \\
\hline $\mathrm{P} 2^{\dagger}$ & 62 & $\mathrm{P} 2 \mathrm{Ex} 4 \mathrm{~F}$ & GTGCCRGCMGGTGGTGCTGT & 71.2 & $\mathrm{P} 2 \mathrm{Ex} 5 \mathrm{R}$ & AATCCCATGTCRTCATCRGA & 60.3 \\
\hline $\mathrm{S} 2^{\dagger}$ & 63 & S2ExAF & GACGCCGGTGGTAGAGGAGG & 70.2 & S2ExBR & GACTTGATCTTCATGTCCTT & 55.5 \\
\hline $\mathrm{S} 2^{\dagger}$ & 64 & $\mathrm{~S} 2 \mathrm{ExBF}$ & AAGGACATGAAGATCAAGTC & 55.5 & S2ExCR & TGGACAGGCATGATCTTCAG & 63.9 \\
\hline $\mathrm{S} 2^{\dagger}$ & 65 & $\mathrm{~S} 2 \mathrm{ExCF}$ & CTGAAGATCATGCCTGTCCA & 63.9 & S2ExDR & ACYTCTTTRGAGCACTTCAC & 52.6 \\
\hline $\mathrm{S} 2^{\dagger}$ & 66 & S2ExDF & CTGCTSATGATGGCTGGTAT & 62.7 & S2ExER & TTCCAGAGATCAGGGGTCAG & 64.2 \\
\hline $\mathrm{S} 3^{\dagger}$ & 67 & S3Ex1F & AAGATGGCGGTGCAAATCTC & 66.2 & S3Ex2R & AACTCRTTCAGCTCGGCYTTG & 65.4 \\
\hline $\mathrm{S} 3^{\dagger}$ & $68 *$ & S3Ex2F & GAGGTRCGTGTGACWCCAAC & 60.5 & S3Ex3R & TTCTCTCCСAGMACATTCTG & 59.4 \\
\hline $\mathrm{S} 3^{\dagger}$ & 69 & S3Ex3F & TGACCGCTGTGGTYCAGAAG & 66.5 & $\mathrm{~S} 3 \mathrm{Ex} 4 \mathrm{R}$ & AGACCACGAGTSGCWACCTT & 66.1 \\
\hline $\mathrm{S} 3^{\dagger}$ & 70 & $\mathrm{~S} 3 \mathrm{Ex} 4 \mathrm{~F}$ & GCAGAGTCTYTGCGYTACAA & 60.6 & S3Ex $5 R$ & AACCKCAGMACACCATAGCA & 59.7 \\
\hline $\mathrm{S} 3^{\dagger}$ & 71 & S3Ex5F & GATGATCCACAGYGGAGACC & 62.0 & S3Ex6R & AGCATGATCTTMACCTTGAT & 55.2 \\
\hline $\mathrm{S} 4^{\dagger}$ & 72 & S4Ex2F & CATTGGATGCTKGACAAACT & 60.1 & S4Ex3R & AYTCCCTCAGYTTGTGGGGA & 65.5 \\
\hline $\mathrm{S} 4^{\dagger}$ & 73 & S4Ex3F & ATCAARATYGATGGCAAGGT & 60.7 & S4Ex4R & ACCWGTYTTCTCGATGCTGA & 62.4 \\
\hline $\mathrm{S} 4^{\dagger}$ & 74 & S4Ex4F & TTTRCWGTTCACCGCATCAC & 61.2 & S4Ex5R & TCRGGGTARCGGATGGTGCG & 70.2 \\
\hline $\mathrm{S} 4^{\dagger}$ & 75 & S4Ex5F & ATYACAGACTTCATCAAGTT & 49.9 & S4Ex6R & TCYCTGTTGGTGATSACACC & 61.2 \\
\hline $\mathrm{S} 4^{\dagger}$ & 76 & S4Ex6F & GGTGTSATCACCAACAGRGA & 61.2 & S4Ex7R & AGGGACACCCAYGGYTTGTT & 65.3 \\
\hline$S 6^{\dagger}$ & $77 *$ & S6Ex2F & GAGAAGCGYATGGCCACAGA & 65.2 & S6Ex3R & ACACCCTGCTTCATGGGGAA & 68.8 \\
\hline$S 6^{\dagger}$ & 78 & S6Ex3F & CGCCAACCTSAGYGTTCTCA & 66.5 & S6Ex4R & AGCCCRGGAATRTCCTTCTC & 61.0 \\
\hline$S 6^{\dagger}$ & 79 & S6Ex4F & ATCCGCAARCTYTTCAACCT & 62.3 & S6Ex5R & GTAACMAGACGCTGAATCTT & 56.0 \\
\hline $\mathrm{S} 6^{\dagger}$ & 80 & S6Ex5F & CCMCGTGTSCTGCAGCACAA & 71.0 & S6Ex6R & TGGCGYTTCTCCTTRGCCTC & 65.4 \\
\hline $\mathrm{S} 7^{\dagger}$ & 81 & S7Ex1F & TGGCCTCTTCCTTGGCCGTC & 72.8 & S7Ex2R & AACTCRTCTGGCTTYTCGCC & 65.3 \\
\hline $\mathrm{S} 7^{\dagger}$ & 82 & S7Ex2F & AGCGCBAAAATAGTGAAGCC & 60.0 & S7Ex3R & GCCTTCAGGTCAGAGTTCAT & 60.8 \\
\hline $\mathrm{S} 7^{\dagger}$ & 83 & S7Ex3F & ATGAACTCTGACCTGAAGGC & 60.8 & S7Ex4R & TGAGGAACRGGMACAAAGAT & 58.7 \\
\hline $\mathrm{S} 7^{\dagger}$ & 84 & S7Ex4F & GTGCGCGAGYTGGARAAGAA & 67.4 & S7Ex5R & TTTGTGCGGCTTTTYCTTGT & 64.4 \\
\hline $\mathrm{S} 7^{\dagger}$ & 85 & S7Ex 5F & AACAAGCARAAGCGTCCYAG & 61.0 & S7Ex6R & CTGGGRAAGACCAGRTCCTC & 62.6 \\
\hline $\mathrm{S} 7^{\dagger}$ & $86^{*}$ & S7Ex6F & CAGAACAAYGTTGAACACAA & 57.0 & S7Ex7R & TGRAACTCTGGGAATTCAAA & 57.1 \\
\hline $\mathrm{S} 8^{\dagger}$ & 87 & S8Ex1F & ACTCTTTCYAGCCRGCGCC & 63.5 & S8Ex2R & CGGTCYTGCGGCGTTTRTGC & 72.5 \\
\hline $\mathrm{S} 8^{\dagger}$ & 88 & S8Ex2F & ACAAGAARAGRAAGTATGAG & 50.0 & S8Ex3R & AGRGCACGGTAYTTCTTGTT & 57.9 \\
\hline $\mathrm{S} 8^{\dagger}$ & 89 & S8Ex3F & AAGAARTACCGTGCTYTGAG & 58.4 & S8Ex4R & TAGACCACATCRATGATCCT & 53.3 \\
\hline $\mathrm{S} 8^{\dagger}$ & $90 *$ & S8Ex4F & GGCMGSAAGAAGGGAGCCAA & 70.8 & S8Ex5R & TGCWGGAACTGCTCCTCCAG & 68.0 \\
\hline $\mathrm{S} 8^{\dagger}$ & 91 & S8Ex5F & GAGGAGCAGTTCCWGCAGGG & 68.5 & S8Ex6R & CCRTCTGCTCKGCCGCACTG & 71.7 \\
\hline $\mathrm{S} 9^{\dagger}$ & 92 & S9Ex2F & GAGAAGTCYCGTCTYGACCA & 59.5 & S9Ex3R & AGGGTGAAYTTSACCCTCCA & 65.5 \\
\hline$S 9^{\dagger}$ & 93 & S9Ex3F & ACCCYAAGCGTCTSTTTGAA & 60.3 & S9Ex4R & CCKCACCAGACGCCTGAGCA & 71.3 \\
\hline $\mathrm{S} 9^{\dagger}$ & $94 *$ & S9Ex4F & GCCAAGAGYATCCACCAYGC & 64.4 & S9Ex 5R & GGGGATGTTCAMACCTGCTT & 65.0 \\
\hline $\mathrm{S} 10^{\dagger}$ & 95 & S10ExAF & CCYGAGCTYGCTGACAAGAA & 63.3 & S10ExBR & CAGGCAAAYTGCTCYTTGAC & 62.3 \\
\hline $\mathrm{S} 12$ & 96 & $\mathrm{~S} 12 \mathrm{Ex} 2 \mathrm{~F}$ & TCTACAATGGCCGAGGAAGG & 66.1 & S12Ex3R & TCATGGATGAGKGCRGTCTT & 60.9 \\
\hline $\mathrm{S} 12$ & 97 & S12Ex3F & ACCGCACTCATCCAYGAYGG & 68.3 & S12Ex4R & CACARAGATGRGCTTGGCGC & 68.1 \\
\hline
\end{tabular}


Table 1 continued

\begin{tabular}{|c|c|c|c|c|c|c|c|}
\hline RP & air & orward & $5^{\prime}-3^{\prime}$ & $\mathrm{Tm}$ & Reverse & $5^{\prime}-3^{\prime}$ & $\mathrm{Tm}$ \\
\hline 12 & 8 & $12 \mathrm{Ex} 4 \mathrm{~F}$ & ATGTYAAGYTGGTGGAGGC & 59.0 & S12Ex5R & AGYTTCTTRTTGTCATCAAC & \\
\hline 12 & 99 & $2 \mathrm{Ex} 5 \mathrm{~F}$ & CGCAAAGTKGTSGGCTGCAG & 71.3 & S12Ex6R & ССТYATTTCTTGGATTTGAA & \\
\hline 15 & 100 & S13ExAF & AGAAAGGGCTTGTCCCAGTC & 64.4 & S13ExBR & ATCTGCTCYTTRACATCATC & \\
\hline 15 & 101 & S13ExBF & GAGCAGATCTTYAARCTGGC & 58.1 & S13ExCR & ACCRGTGACGAARCGCACCT & \\
\hline $13^{\dagger}$ & 102 & 13ExCF & TACCACCTMATCAAGAAGGC & 58.2 & S13ExDR & AGAATCAGGCGGAAYTTGGC & \\
\hline $14^{\dagger}$ & 103 & $14 \mathrm{Ex} 2 \mathrm{~F}$ & TTCGCMTCCTTCAACGACAC & 66.1 & S14Ex3R & TCGGCCTTYACCTTCATCCC & \\
\hline $14^{\dagger}$ & 104 & $14 \mathrm{Ex} 3 \mathrm{~F}$ & ATCACWGCKCTGCACATCAA & 62.1 & S14Ex4R & CCWGGTCCAGGRGTCTTGGT & \\
\hline $14^{\dagger}$ & 105 & $14 \mathrm{Ex} 4 \mathrm{~F}$ & GGGGCMCAGTCKGCCCTCAG & 71.7 & S14Ex5R & GTCACCCCGATCCCGTCAGA & \\
\hline & 106 & $\mathrm{Ex} 2 \mathrm{~F}$ & GACCAGCTKCTGGACATGTC & 62.4 & Ex3R & GGMGCCTCCTTCT" & \\
\hline & 107 & & GGCAAGACYTTCAACCAGGT & 63. & & GCTTGTARG & \\
\hline & & & & & & & \\
\hline & & & & & & & \\
\hline & & & & & & & \\
\hline & & & & & & & \\
\hline & & & & & & & \\
\hline & & & & & & & \\
\hline & & & CTGTGCGYATGCCYACCAAG & & & GTGATCTGCTTRACRATCTC & \\
\hline & & $\mathrm{Ex} 2 \mathrm{~F}$ & GARTTCGTGGACCTG & & & ATRGAGGCRTGGTCCTTGGC & \\
\hline & 116 & Ex3F & GACCAYGCCTCYATCCAGAT & 60.5 & Ex4R & GTCTTGAACTGKCCATTRAA & \\
\hline & 117 & $1 \mathrm{Ex} 4 \mathrm{~F}$ & TTCAAGACCTAYGCYATCTG & 54.4 & S21Ex5R & GMCACRATGCTGTCGGTCTT & \\
\hline $24^{\dagger}$ & 118 & $\mathrm{~S} 24 \mathrm{Ex} 2 \mathrm{~F}$ & TGCTTCAGAGGAAGCAAATG & 63.0 & S24Ex3R & ATGTACAARACCACCCCTGA & \\
\hline $24^{\dagger}$ & 119 & S24Ex3F & GCMAAGAARAATGAGCCCAA & 63.8 & S24Ex4R & TTCATTCTGTTCTTGCGTTC & \\
\hline $25^{\dagger}$ & 120 & S25ExAF & AARKCCAAAAAGGACAAGGA & 59.9 & S25ExBR & TCGAAGAGGACCAGGTTGTT & \\
\hline $\mathrm{S} 25^{\dagger}$ & 121 & $\mathrm{~S} 25 \mathrm{ExBF}$ & ATCCGDGGCTCYCTGGCCAG & 70.1 & S25ExCR & TTTYGACACCARTTTGATCA & 58 \\
\hline S26 & 122 & $\mathrm{~S} 26 \mathrm{Ex} 2 \mathrm{~F}$ & ATCAGGAAYATWGTGGAGGC & 57.1 & S26Ex3R & ACACAGTARTGYAGCTTCAC & 1 \\
\hline S28 & 123 & $\mathrm{~S} 28 \mathrm{Ex} 1 \mathrm{~F}$ & GATGCCAGYCGYGTGCAGCC & 71.7 & S28Ex2R & CCCTGRGARCCAGTTCTTCC & 2. \\
\hline S28 & 124 & S28Ex2F & GGAAGAACTGGYTCYCAGGG & 62.6 & & ATGATKGAKCGGTTGCTGTC & 2 \\
\hline S29 & 125 & S29Ex1F & CAGCAGCTCTAYTGGAGYCA & 60.7 & S29Ex2R & CACTGRCGGCACATRTTGAG & 1 \\
\hline S30 & 126 & $\mathrm{~S} 30 \mathrm{Ex} 2 \mathrm{~F}$ & CACACCCTTGAGGTGACSGG & & S30Ex3R & CCCTCYAGATYCTGGACATG & 8 \\
\hline S30 & 127 & & TCAGAGYACTGCACYCTGGA & 58.7 & $\mathrm{~S} 30 \mathrm{Ex} 4 \mathrm{R}$ & ACTTTTCCRGCACGRGCCAG & \\
\hline $\mathrm{S} 30$ & 128 & $\mathrm{~S} 30 \mathrm{Ex} 4 \mathrm{~F}$ & CTGGCYCGTGCYGGAAAAGT & 66.6 & S30Ex5R & TTGGCRCGRCCAGTCTTCTT & 64 \\
\hline
\end{tabular}

$\uparrow$ Genes found in the draft genome sequences of the Pacific bluefin tuna (Thunnus orientalis)

* Selected primer pairs to investigate universal specificity

found in the draft genome sequences of the Pacific bluefin tuna. Twelve primer pairs (Table 1, asterisk) expected to amplify 500-1000 bp fragments in the Pacific bluefin tuna were selected, and the PCR amplification results are shown in Fig. 1 and summarized in Table 2. All amplified fragments were larger than those expected for intronless amplicons when separated using agarose gel electrophoresis. Amplified fragments were observed for all primer pairs in the Japanese puffer (lane 2), starry flounder (lane 3), and the Pacific bluefin tuna (lane 4), whereas no amplification was observed for primer pair 17 in the Japanese eel (lane 6), for primer pair 86 in broadbanded thornyhead (lane 5), and for three primer pairs (17, 68, and 86) in the Japanese pilchard (lane 1). The Orders to which the Japanese eel, broadbanded thornyhead, and the Japanese pilchard belonged were not involved in primer design, which may cause the slight difference in amplification success between the former and later fish groups. The highest score for single fragment amplification was observed in starry flounder ( 10 of 12 primer pairs) and the lowest was observed in the Pacific bluefin tuna and broadbanded thornyhead (6 of 12 primer pairs), whereas the total score for single fragment amplification was relatively high $(62.5 \%)$ (45 of 72 primer pairs). Clear or nearly two-band PCR products were observed for primer pair 26 in Japanese pilchard and Pacific bluefin tuna, for primer pair 38 in Japanese eel, for primer pair 49 in starry 

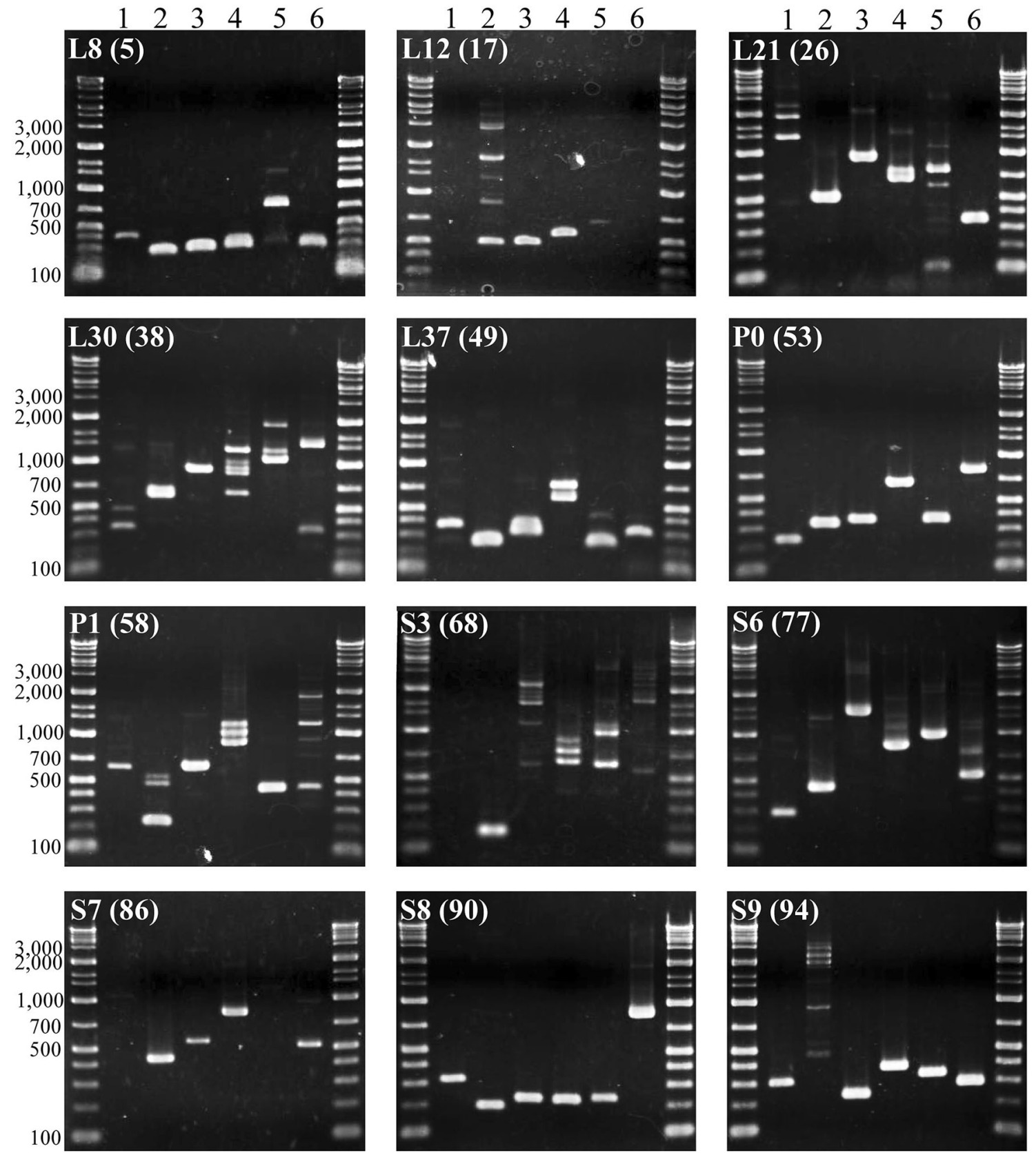

Fig. 1 Agarose gel electrophoresis images of the PCR products amplified using 12 selected primer pairs for ribosomal protein gene (RP) introns. RP genes are shown at the top-left in each gel image and the primer pair number is shown in parenthesis (see also Table 1). The left and right most ends in each gel are the size marker, and the 1st to 6th lanes are the Japanese pilchard, the Japanese puffer, starry flounder, the Pacific bluefin tuna, broadbanded thornyhead, and the Japanese eel, respectively

flounder and Pacific bluefin tuna, and for primer pair 68 in Pacific bluefin tuna and broadbanded thornyhead. These could be heterozygotes and potential candidates for population genetic analysis. Amplification of three or more fragments was relatively fewer incidence (12 of 72 primer pairs), which may be attributable to paralogs, pseudogenes, and/or non-specific annealing. Elevation of annealing temperature may decrease number of amplified fragments as demonstrated previously (Chow et al. 2015). The sizes of amplified fragments of Pacific bluefin bluefin tuna using nine primer pairs (Table 2, dagger) corresponded to those expected from the genomic data, whereas fragments amplified using three primer pairs $(5,90$, and 94) were shorter than 
Table 2 Number of fragments amplified by 12 primer pairs in six fish species

\begin{tabular}{|c|c|c|c|c|c|c|c|c|c|}
\hline $\mathrm{RP}^{*}$ & PB & IN & EX & Pilchard & Puffer & Flounder & Tuna & Rockfish & Eel \\
\hline L8 (5) & 795 & 605 & 190 & 1 & 1 & 1 & 1 & 3 & 1 \\
\hline L12 (17) & $573^{\dagger}$ & 433 & 140 & 0 & $5+$ & 1 & 1 & 1 & 0 \\
\hline L21 (26) & $661^{\dagger}$ & 566 & 95 & $2+$ & 1 & 1 & 2 & $4+$ & 1 \\
\hline L30 (38) & $935^{\dagger}$ & 843 & 92 & 2 & 1 & 1 & $5+$ & 3 & 2 \\
\hline L37 (49) & $725^{\dagger}$ & 657 & 68 & 1 & 1 & 2 & 2 & 2 & 1 \\
\hline P0 (53) & $658^{\dagger}$ & 548 & 110 & 1 & 1 & 1 & 1 & 1 & 1 \\
\hline P1 (58) & $985^{\dagger}$ & 887 & 98 & 1 & 3 & 1 & 3 & 1 & $3+$ \\
\hline S3 (68) & $736^{\dagger}$ & 662 & 74 & 0 & 1 & $4+$ & 3 & 3 & $2+$ \\
\hline S6 (77) & $857^{\dagger}$ & 741 & 116 & 1 & 1 & 1 & $2+$ & 1 & $2+$ \\
\hline S7 (86) & $825^{\dagger}$ & 733 & 92 & 0 & 1 & 1 & 1 & 0 & 1 \\
\hline S8 (90) & 693 & 553 & 140 & 1 & 1 & 1 & 1 & 1 & 1 \\
\hline S9 (94) & 600 & 522 & 78 & 1 & $5+$ & 1 & 1 & 1 & 1 \\
\hline
\end{tabular}

Pilchard (Sardinops melanostictus), puffer (Takifugu rubripes), tuna (Thunnus orientalis), rockfish (=broadbanded thornyhead) (Sebastolobus macrochir), and eel (Anguilla japonica)

$P B$ total fragment size expected for Pacific bluefin tuna (Thunnus orientalis), IN intron size expected for Pacific bluefin tuna, $E X$ expected fragment size for intron less amplification

* Target ribosome protein gene and primer pair number (in parenthesis) used

$\uparrow$ Size of fragment amplified was corresponding to that expected from genomic data of Pacific bluefin tuna

expected, indicating that these three primer pairs annealed and amplified non-target regions of the Pacific bluefin tuna genome.

Although nucleotide sequence analysis may be necessary to characterize and authenticate the amplicons of interest, the present study offers new sets of nuclear primers that are potentially applicable to wide variety of fish taxa and can be an initial step toward isolating single-copy nuclear DNA sequences. Subsequent polymorphism detection may also provide a more informative database for genetic species identification and population studies.

Acknowledgments This work was partially supported by a Grant-in-Aid for Scientific Research on Priority Areas (C) (No. 25450292) from the Ministry of Education, Science, Sports, and Culture of Japan.

Open Access This article is distributed under the terms of the Creative Commons Attribution 4.0 International License (http:// creativecommons.org/licenses/by/4.0/), which permits unrestricted use, distribution, and reproduction in any medium, provided you give appropriate credit to the original author(s) and the source, provide a link to the Creative Commons license, and indicate if changes were made.

\section{References}

Atarhouch T, Rami M, Naciri M, Dakkak A (2007) Genetic population structure of sardine (Sardina pilchardus) off Morocco detected with intron polymorphism (EPIC-PCR). Mar Biol 150:521-528

Berrebi P, Boissin E, Fang F, Cattaneo-Berrebi G (2005) Intron polymorphism (EPIC-PCR) reveals phylogeographic structure of Zacco platypus in China: a possible target for aquaculture development. Heredity 94:589-598

Chow S (1998) Universal PCR primer for calmodulin gene intron in fish. Fish Sci 64:999-1000

Chow S, Hazama K (1998) Universal PCR primers for S7 ribosomal protein gene introns in fish. Mol Ecol 7:1255-1256

Chow S, Takeyama H (2000) Nuclear and mitochondrial DNA analyses reveal four genetically separated breeding units of the swordfish (Xiphias gladius). J Fish Biol 56:1087-1098

Chow S, Nakadate M (2004) PCR primers for fish G6PD gene intron and characterization of intron length variation in the albacore Thunnuns alalunga. Mol Ecol Notes 4:391-393

Chow S, Clarke S, Nakadate M, Okazaki M (2007) Boundary between the north and south Atlantic populations of the swordfish (Xiphias gladius) inferred by a single nucleotide polymorphism at calmodulin gene intron. Mar Biol 152:87-93

Chow S, Yanagimoto T, Nakamura Y (2015) Universal primers for exon-priming intron-crossing (EPIC) PCR on ribosomal protein genes in marine animals. Int $\mathrm{J}$ Aquat Res 7:245-250

Corte-Real HBSM, Dixon DR, Holland PWH (1994) Intron-targeted PCR: a new approach to survey neutral DNA polymorphism in bivalve populations. Mar Biol 120:407-413 
Creer S (2007) Choosing and using introns in molecular phylogenetics. Evol Bioinform 3:99-108

Daguin C, Bonhomme F, Borsa P (2001) The zone of sympatry and hybridization of Mytilus edulis and M. galloprovincialis, as described by intron length polymorphism at locus mac-1. Heredity $86: 342-354$

DeBry R, Seshadri S (2001) Nuclear intron sequences for phylogenetics of closely related mammals: an example using the phylogeny of Mus. J Mammal 82:280-288

Hassan M, Lemaire C, Fauvelot C, Bonhomme F (2002) Seventeen new exon-primed intron crossing polymerase chain reaction amplifiable introns in fish. Mol Ecol Notes 2:334-340

He S, Mayden RL, Wang X, Wang W, Tang KL, Chen WJ, Chen Y (2008) Molecular phylogenetics of the family Cyprinidae (Actinopterygii: cypriniformes) as evidences by sequence variation in the first intron of S7 ribosomal protein-coding gene: further evidence from a nuclear gene of the systematic chaos in the family. Mol Phylogenet Evol 46:818-829

Jarman SN, Ward RD, Elliot NG (2002) Oligonucleotide primers for PCR amplification of coelomate introns. Mar Biotechnol 4:347-355

Jennings RM, Etter RJ (2011) Exon-primed, intron-crossing (EPIC) loci for five nuclear genes in deep-sea protobranch bivalves: primer design, PCR protocols and locus utility. Mol Ecol Resour 11:1102-1112

Kenmochi N, Kawaguchi T, Rozen S, Davis E, Goodman N, Hudson TJ, Tanaka T, Page DC (1998) A map of 75 human ribosomal protein genes. Genome Res 8:509-523

Lessa EP (1992) Rapid survey of DNA sequence variation in natural populations. Mol Biol Evol 9:323-330

Nakadate M, Chow S (2008) Isolation and characterization of single copy nuclear DNA markers in the Atlantic bluefin tuna Thunnus thynnus. Fish Sci 74:1333-1335

Nakadate M, Viñas J, Corriero A, Clarke S, Suzuki N, Chow S (2005) Genetic isolation between Atlantic and Mediterranean albacore (Thunnus alalunga) populations inferred from mitochondrial and nuclear DNA markers. J Fish Biol 66:1545-1557

Nakamura Y, Mori K, Saitoh K, Oshima K, Mekuchi M, Sugaya T, Shigenobu Y, Ojima N, Muta S, Fujiwara A, Yasuike M, Oohara I, Hirakawa H, Chowdhury VS, Kobayashi T, Nakajima K, Sano M, Wada T, Tashiro K, Ikeo K, Hattori M, Kuhara S, Gojobori T, Inoue K (2013) Evolutionary changes of multiple visual pigment genes in the complete genome of Pacific bluefin tuna. PNAS 110:11061-11066

Near TJ, Cheng CHC (2008) Phylogenetics of notothenioid fishes (Teleostei: Acanthomorpha): inferences from mitochondrial and nuclear gene sequences. Mol Phylogenet Evol 47:832-840

Nohara K, Okamura H, Nakadate M, Hiramatsu K, Suzuki N, Okazaki M, Chow S (2003) Biological investigation on two types of bill internal structure of swordfish (Xiphias gladius) and genetic differentiation between the North and South Atlantic stocks. Bull Fish Res Agency 7:1-13 (in Japanese with English abstract)

Pacheco NM, Congdon BC, Friesen VL (2002) The utility of nuclear introns for investigating hybridization and genetic introgression: a case study involving Brachyramphus murrelets. Conserv Genet 3:175-182

Palumbi SR, Baker CS (1994) Contrasting population structure from nuclear intron sequences and mtDNA of humpback whales. Mol Biol Evol 11:426-435

Pinho C, Rocha S, Carvalho BM, Lopes S, Mourão S, Vallinoto M, Brunes T, Haddad CFB, Gonçalves H, Sequeira F, Ferrand N (2010) New primers for the amplification and sequencing of nuclear loci in a taxonomically wide set of reptiles and amphibians. Conserv Genet Resour 2:181-185

Quattro JM, Jones WJ (1999) Amplification primers that target locus-specific introns in actinopterygian fishes. Copeia 1:191-196

Thompson JD, Gibson TJ, Plewniak F, Jeanmougin F, Higgins DG (1997) The CLUSTALX windows interface: flexible strategies for multiple sequence alignment aided by quality analysis tools. Nucleic Acids Res 25:4876-4882

Touriya A, Rami M, Cattaneo-Berrebi G, Ibanez C, Augros S, Boissin E, Dakkak A, Berrebi P (2003) Primers for EPIC amplification of intron sequences for fish and other vertebrate population genetic studies. Biotechniques 35:676-682

Williams ST, Jara J, Gomez E, Knowlton N (2002) The marine Indo-west Pacific break: contrasting the resolving power of mitochondrial and nuclear genes. Integr Comp Biol 42:941-952

Wool IG (1979) The structure and function of eukaryotic ribosomes. Annu Rev Biochem 48:719-754

Wool IG, Chan Y-L, Gluck A (1995) Structure and evolution of mammalian ribosomal proteins. Biochem Cell Biol 73:933-947

Yanagimoto T, Kitamura T, Kobayashi T (2012) Genetic stock structure of walleye Pollock (Theragra chalcogramma) inferred by PCR-RFLP analysis of the mitochondrial DNA and SNP analysis of nuclear DNA. Mar Genomics 7:17-25

Yao X, Liu L, Yan M, Li D, Zhong C, Huang H (2015) Exon primed intron-crossing (EPIC) markers reveal natural hybridization and introgression in Actinidia (Actinidiaceae) with sympatric distribution. Biochem Syst Ecol 59:246-255

Yoshihama M, Uechi T, Asakawa S, Kawasaki K, Kato S, Higa S, Maeda N, Minoshima S, Tanaka T, Shimizu N, Kenmochi N (2002) The human ribosomal protein genes: sequencing and comparative analysis of 73 genes. Genome Res 12:379-390 